\title{
Is diagnosing patients with Organic Acidurias and Aminoacidopathies enough? Conundrums of a low middle-income country
}

\author{
Hafsa Majid', Lena Jafri², Zeba Zulfiqar Ali ${ }^{3}$, Bushra Afroze ${ }^{4}$
}

\begin{abstract}
Objective: This study was done to determine the factors responsible for non-treatment of inherited metabolic disorders (IMDs) requiring food for special medical purposes (FSMPs) in Pakistan.

Methods: A descriptive cross-sectional study was conducted by Departments of Pediatrics \& Child Health and Pathology \& Laboratory Medicine, Aga Khan University. Patients diagnosed with IMDs from January 2013 to December 2016 requiring FSMPs were surveyed after a year of initial diagnosis to collect the details of treatment advised, mortality status, and reasons of non-treatment, including not prescribed by physician, non-acceptance by family, non-availability or non-affordability.

Results: Over four years period, 311 patients were identified with IMDs; Median age of patients was 1.0 yrs $(0.0 .2-3.65)$ with $54 \%(n=168)$ being male. Of the total $38.2 \%(n=119)$ required FSMPs, $9 \%(n=28)$ patients were excluded due to unavailability of diagnostics information. Parents of 58 patients requiring FSMPs out of 119 participated in survey. The leading causes of non-treatment were, FSMPs not prescribed by physicians $(n=30,51.7 \%)$ followed by non-affordability $(n=23,39.6 \%)$, families' unacceptance in $(n=9,18 \%)$ patients, non-availability of FSMPs $(n=2,3.4 \%)$ and early death of patient $(n=1,1.7 \%)$.

Conclusion: The main factors responsible for non-treatment of FSMPs requiring IMDs were non-prescription by physician and non-affordability.
\end{abstract}

KEYWORDS: Inherited metabolic disorder, Pakistan, Survey, Treatment, non-prescription, non-affordability, food for special medical purposes.

doi: https://doi.org/10.12669/pjms.37.7.3887

How to cite this:

Majid H, Jafri L, Ali ZZ, Afroze B. Is diagnosing patients with Organic Acidurias and Aminoacidopathies enough? Conundrums of a low middle-income country. Pak J Med Sci. 2021;37(7):1896-1901. doi: https://doi.org/10.12669/pjms.37.7.3887

This is an Open Access article distributed under the terms of the Creative Commons Attribution License (http://creativecommons.org/licenses/by/3.0), which permits unrestricted use, distribution, and reproduction in any medium, provided the original work is properly cited.

1. Dr. Hafsa Majid, FCPS. Section of Chemical Pathology,

Department of Pathology and Laboratory Medicine,

2. Dr. Lena Jafri, FCPS,

Section of Chemical Pathology,

Department of Pathology and Laboratory Medicine,

3. Zeba Zulfiqar Ali,

Clinical Nurse Coordinator,

Department of Paediatrics \& Child Health.

4. Dr. Bushra Afroze, FCPS,

Department of Paediatrics \& Child Health,

1-4: Aga Khan University, Stadium Road,

P.O. Box 3500. Karachi 74800, Pakistan.

Correspondence:

Dr. Bushra Afroze, FCPS,

Department of Paediatrics \& Child Health,

Aga Khan University. Stadium Road,

P.O. Box 3500, Karachi 74800, Pakistan.

E-mail: bushra.afroze@aku.edu

* Received for Publication:

* $1^{\text {st }}$ Revision Received:

* $2^{\text {nd }}$ Revision Received:

* Final Revision Accepted:
November 5, 2020

January 13, 2021

June 3, 2021

June 15, 2021

\section{INTRODUCTION}

Inherited metabolic disorders (IMDs) encompass a heterogeneous group of genetic diseases due to defective metabolic processes. As defined recently, any condition in which the impairment of a biochemical pathway is intrinsic to the pathophysiology of the disease is considered an IMD. ${ }^{1}$ The cumulative incidence of IMDs varies between one in 1400 and one in $3000 .^{2,3}$ The measured prevalence in a particular country depends on the population selected and the method employed for screening. The prevalence of IMDs is unknown in Pakistan but is expected to be high due to the cultural preference of consanguineous marriages. ${ }^{4,5}$

The IMDs caused by deficiency of enzymes in a metabolic pathway result in accumulation of toxic metabolites and deficiency of essential needed 
metabolites. Effective treatment of a group of IMD depends primarily on dietary restriction because off-the-shelf foods cannot be metabolized by these patients, resulting in toxic effects. ${ }^{6}$ Typically, a healthcare provider prescribes a specialized diet restricting the offending metabolites that cannot be metabolized while monitoring and providing all the other necessary metabolites essential for normal growth and development. ${ }^{4}$ For example, individuals with phenylketonuria are unable to properly metabolize the amino acid phenylalanine, which must be selectively limited in their diet to prevent severe intellectual disability. ${ }^{7,8}$ Disorders of amino acid and fatty acid metabolism require medical foods that restrict the offending amino acid(s) or long-chain fatty acids. ${ }^{9-11}$

Several organic acidemias (OA) and aminoacidopathies are treatable with the good outcome by Food for Special Medical Purposes (FSMPs), which is a life-long necessity for a patient. ${ }^{12,13}$ In 2013, through concerted efforts, FSPMs were made available locally. The local literature on the patient's outcome diagnosed with IMDs, although limited, reports the poor outcome of these patients. Local studies have reported that many of the patients diagnosed with IMDs are not on treatment. There is a need to identify the issues leading to the non-treatment of these patients, design and implement strategies to overcome these issues and improve patient outcomes.

This study was conducted to obtain an up-todate estimate of the outcomes of patients with IMDs requiring FSMPs treatment in the Pakistani population and determine the factors responsible for non-treatment of IMDs requiring FSMPs.

\section{METHODS}

This descriptive cross-sectional study was conducted by the Departments of Pediatrics and Child Health and Pathology \& Laboratory Medicine. Data of patients diagnosed with organic acidemias and aminoacidopathies based on urine organic acid and plasma amino acid testing from January 2013 to December 2016 were included in this study using a non-probability consecutive sampling technique. The treating physicians in different cities were informed of the diagnosis by telephone or email by a Chemical Pathologist about their patients' diagnosis. Only organic acidurias and aminoacidopathies requiring FSMPs were included in this study, while those requiring orphan drugs for treatment were excluded. The fatty acid oxidation defects were not included in the present study, as they cannot be diagnosed on urine organic and amino acids analysis.

Grouping of Inherited Metabolic Disorders: Organic acidemias and aminoacidopathies were categorized into two groups; Decompensation Group, which present with metabolic decompensation and encephalopathy and may lead to death when untreated. These include methylmalonic academia (MMA), urea cycle disorders (UCD), maple syrup urine disease (MSUD), Isovaleric academia (IVA), propionic acidemia (PA), glutaric aciduria type 1 (GA-1). The second group, the Non-Decompensation group, does not present with metabolic decompensation. The clinical course is rather static with intellectual disability and neurological impairment rather than mortality if not treated properly. These include phenylketonuria (PKU) \& cystathionine beta-synthase (CBS) deficiency. Since it was a telephonic survey, intellectual disability and neurological impairment was not included as it requires formal assessment using structured ageappropriate tools by a trained clinical psychologist or neuro-developmental specialist.

Survey to identify factors leading to treatment non-initiation: Only patients diagnosed with either organic acidemia or amino acidopathies for more than one year were included, and the survey was prospectively conducted from Jan to March 2018. Parents or guardian of patients were surveyed via telephone by the metabolic nurse in 10 to 15 minutes. Metabolic nurses are specialized clinical nurse coordinators trained in dealing with patients with IMDs, who have been working with a metabolic physician for the last four years. The principal investigator reviewed data collected by the metabolic nurse to identify missing data, and parents/guardian were contacted again to gather that missed data.

Details of treatment advised by the primary physicians, mortality status, and treatment not initiated or discontinued, reasons of non-treatment including not prescribed by the physician, nonacceptance by family, non-availability of FSMPs or non-affordability were collected.

Outcome assessment of patients with decompensation group of IMDs: Patients included in the decompensation group of IMDs were also surveyed to gather data about their outcome in the form of mortality. Patients were categorized into two groups based on the healthcare provider's speciality, i.e., under the care of a general pediatrician (practising all over Pakistan) or metabolic physicians (housed at AKUH only). 
Ethical Consideration: The study was done per Helsinki's ethical code. In order to maintain confidentiality, coding was given to patients, and their original identifications were removed. An exemption was sought from Institution's Ethical Review Committee (ERC number: Ped-5144)

Statistical Analysis: The statistical analysis was performed using the statistical package social sciences version 19. Frequency and percentages were generated for categorical variables and median with interquartile ranges (IQR) for quantitative variables. A Chi-square test was performed to compare the outcome of patients under the care of general pediatricians and metabolic physicians.

\section{RESULTS}

Patients tested for OA and aminoacidopathies during the study period were 2499. Total 311 patients were tested positive for IMD. Median (Q3Q1) age of patients was one years (0.0.2-3.65) with $54 \%(n=168)$ being male. Of the total $38.2 \%(n=119$ of 311) required FSMPs for treatment, $52.7 \%(n=164)$ were in the non-FSMP treated group and $9 \%(n=28)$ patients were excluded due to non-availability of complete clinical and diagnostic information. The treatment status of patients is shown in Fig.1.
Grouping of Inherited Metabolic Disorders: In the FSMPs treatment group, $68 \%(n=81$ of 119$)$ belonged to the decompensation group of IMDs, and 31.9\% $(n=38$ of 119$)$ were in the non-decompensation group. In the decompensation group, $34.4 \%(n=41$ of 119 ) were prescribed FSMPs, and $20.1 \%$ ( $n=24$ of 119) of these took it, while the rest either did not take or discontinued FSMPs after some time, Fig.1. In the non-decompensation IMD group 14.3\% $(\mathrm{n}=17)$ patients, were prescribed FSMPs, of which $2.5 \%(\mathrm{n}=3)$ actually took it.

Survey to identify factors leading to treatment noninitiation or discontinuation: Data analysis was done using the Chi-square test to identify factors leading to non-initiation or discontinuation. Both alive and deceased patients who were prescribed FSMPs treatment were included in this survey; $48.7 \%$ $(n=58$ out of 119) patients. Top three leading causes for the treatment non-initiation or discontinuation were; FSMPs not prescribed by physicians in 51.7\% $(n=30)$ cases followed by non-affordability $39.6 \%$ $(n=23)$ and families unacceptance in $18 \%(n=9)$, shown in Fig.2.

Outcome assessment of patients with decompensation group of IMDs: Out of the total 119 patients that required FSMP, 68\% ( $n=81$ out

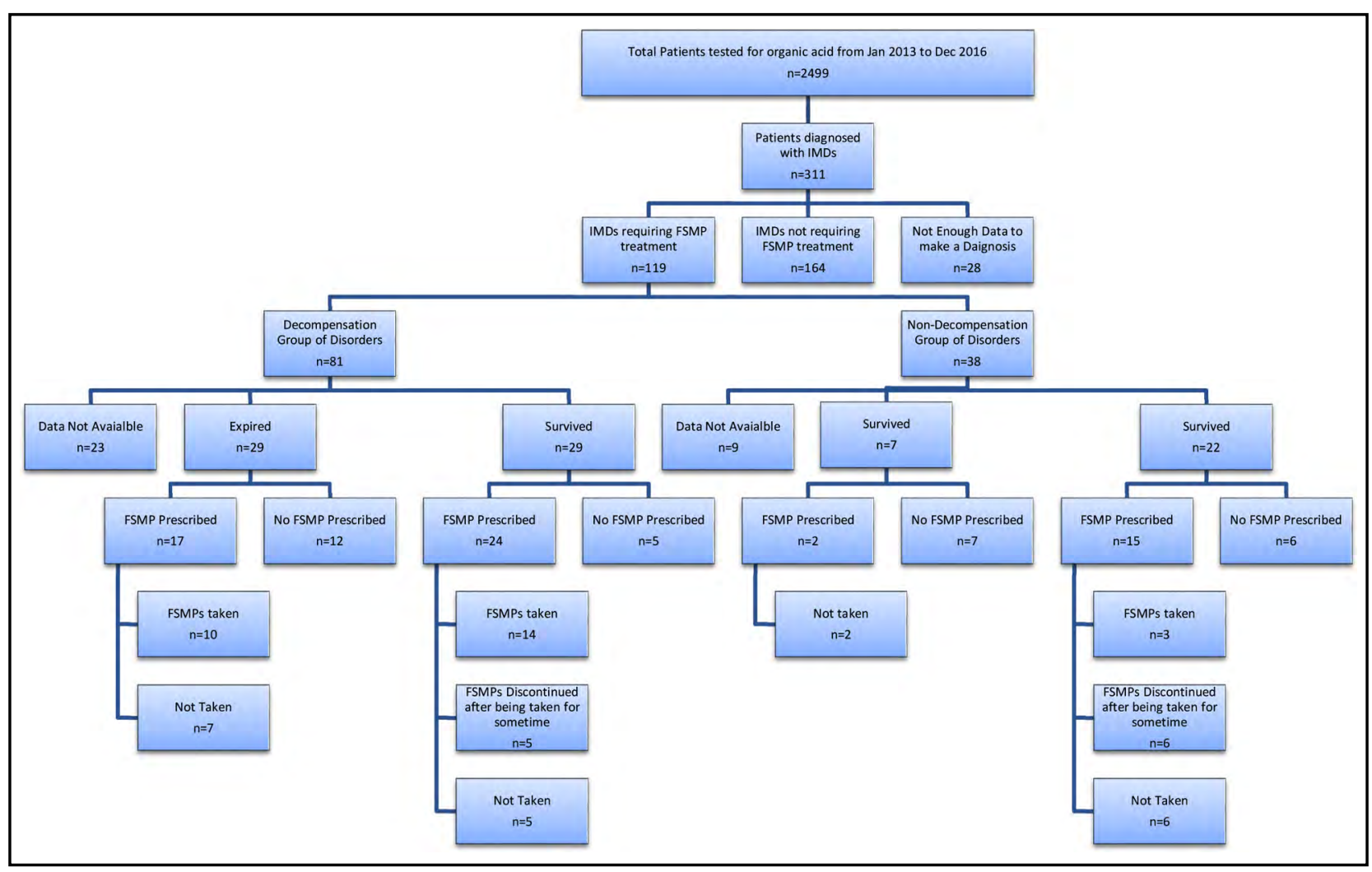

Fig.1: Distribution of study patients based on treatment status. 


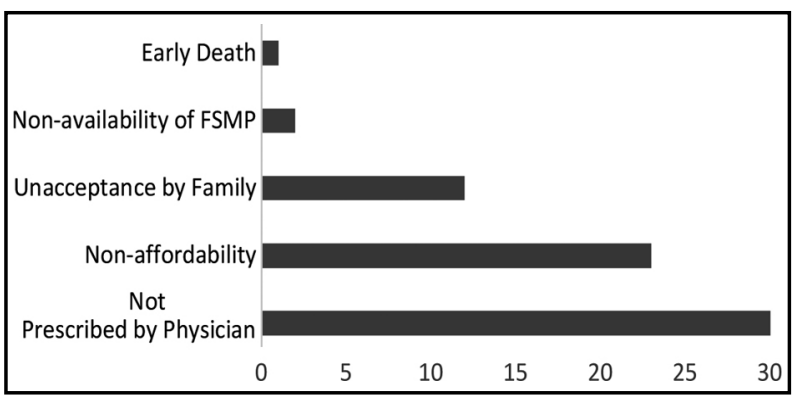

Fig.2: Distribution of factors leading to treatment non-initiation or discontinuation.

The number of responses is greater than the number of respondents because respondents were allowed to choose more than one response.

of 119) had decompensated group IMD. Of the patients with a decompensated group of IMDs, 38\% $(n=31$ out of 81$)$ were under the care of a metabolic physician, while general pediatricians from all over Pakistan followed $62 \%(n=50$ out of 81$)$. Complete information was available for 29 patients in each group. Mortality was higher in patients under the care of general pediatricians than a metabolic physician, $62 \%$ ( $\mathrm{n}=18 / 29$ patient expired) and 38\% $(n=11 / 29$ patient expired). The $\mathrm{p}$-value determined using the chi-square test was 0.0572. While survival was better in patients treated by metabolic physician, $62 \%(n=18 / 29)$ vs. $38 \%(n=11 / 29)$, with a p-value of 0.0572 .

\section{DISCUSSION}

The prevalence of IMDs in our population is high. ${ }^{14-17}$ There is limited local literature available on different IMDs, which are mostly prevalence studies, but none have reported the outcome of IMD patients after diagnosis. ${ }^{14-16,18}$ Awareness regarding prompt diagnosis and early treatment of IMDs is increasing locally. Nevertheless, nearly half the IMD patients diagnosed are not on treatment. Therefore, there is a dire need to identify barrier in IMD patient's management and reduce them. For IMDs requiring FSMPs, dietary therapy is the effective primary treatment. This is the first survey from Pakistan to obtain information about management and factors leading to the non-treatment of IMDs requiring FSMPs. In the present study, only one fifth $(22.6 \%, \mathrm{n}=27$ of 119$)$ patients requiring FSMPs were on appropriate treatment. Similar findings were reported by another study done from our centre during 2008-2012, which reported that only one-third of IMD patients were treated and under regular follow-up. Non-affordability was the main factor for non-compliance, as at that time, the FSMPs

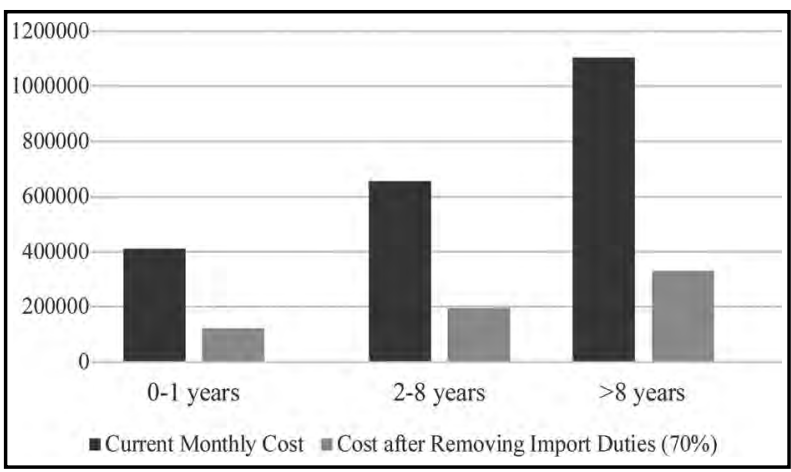

Fig.3: Estimated Annual Treatment Cost (PKR) of FSMP for MSUD in Pakistan, before and after removing the custom duties.

were imported in small batches at a high cost. ${ }^{19}$ The proportion of treatment non-compliance/noninitiation in the present study is almost the same as in the previous study, $51 \%$ vs $57 \%$, respectively.

In the present study, non-prescription by the physicians was one of the major factors for treatment non-initiation. This point towards limited knowledge of general pediatricians regarding the appropriate use of FSMPs. These findings advocate the need for a knowledge assessment survey of the physicians taking care of IMD patients and better system integration. A local study on MMA patients reports similar finding for optimal outcomes. ${ }^{20}$ However, the sustainability of treatment is only possible if the FSMPs cost is more acceptable to families and donors. So, in order of significance, addressing the issue of high FSMPs cost needs to be prioritized. Similar findings were reported by a cross-sectional study done on Pakistani patients with Hyperphenylalaninemia, stating financial constraints as the major cause of treatment non-compliance. ${ }^{21}$

Healthcare infrastructure in Pakistan is very meagre. There is no health insurance system in Pakistan to cover the cost of FSMPs. The private sector provides health care services to almost twothirds of the population, and health expenses are mostly paid out-of-pocket. ${ }^{22}$ The FSMPs are highly regulated, and specialized life-saving products used to treat IMDs, which are manufactured under strict medical vigilance, thus having a generally higher cost than standard infant formulas. Unfortunately, the FSMPs are not produced locally but are exported from other countries. However, the critical difference between the FSMPs as the lifesaving treatment and the regular infant formulas as food supplements is not recognized by the Government of Pakistan. Thus, FSMPs in Pakistan 
are imported under the same Harmonized System code (HS code 2016.9.9) as regular infant formulas. As a result, an accumulative tax of $70 \%$ is applied to this life-saving treatment.

In contrast, the tax duties charged on importing FSMPs in countries like Iran and Turkey (HS Code 3004.5000 ) are $15 \%$ each. Due to the current tax structure, philanthropicagencies hesitatetosupport FSMP treatment as most philanthropic groups wish to transfer the full benefit of every penny donated directly to those in need of the support rather than paying the taxes to governments. Advocacy at the government level to reduce the tax on the FSMPs is critical for sustainable treatment. The impact of high import duties on FSMPs becomes evident when, for example, the treatment cost of MSUD is compared before and after applying custom duties in different age groups (Fig.2). If customs duties are removed, nearly 3.3 patients can be treated with the same amount of money currently spend to treat one patient, as shown in Fig.3.

In western countries, the situation is better due to medical insurance coverage, where medical insurances or other resources either completely or partially covers FSMPs cost. ${ }^{23}$ Locally published literature also reports that the high cost of FSMPs is a major cause of the low treatment compliance of patients. ${ }^{24}$ The high tax on the lifesaving FSMPs needs to be addressed in Pakistan for the patients diagnosed through high-risk screening and to pave the path of the newborn screen for IMDs in Pakistan, which is now the standard of care for all babies born in most of the World.

In the current study, only decompensation group patients were included as the patients with a non-decompensation group of IMDs develop intellectual disability with static clinical course and mortality was measured as the main outcome measure. Patients under the care of general pediatricians had a higher mortality rate than the patients under care of metabolic physicians. This points to the significance of specialized supervision of medical care of IMD patients given by the metabolic physician.

Another gap that needs to be addressed is to develop a good referral system for these patients. In Pakistan, seeking consultation from a specialist physician is mostly the parent's responsibility. Moreover, a lack of timely referral to a metabolic specialist is also an important factor in poor patient outcome. An integrated referral system needs to be developed in Pakistan, and general pediatricians should be educated to use that system appropriately.
Families' reluctance to accept the life-long treatment with various dietary restrictions and the use of FSMPs was also identified as a reason for non-compliance in our study. Many felt that they would be depriving their child of a normal life with life-long dietary restrictions. One patient was started on non-medical treatment in the form of homeopathic therapy. At thesametime, two families gave up treatment only after a few weeks as they found no positive response, while one family did not initiate treatment due to their religious beliefs. These findings highlight the need to create public awareness of IMDs and the importance of longterm compliance to treatment. Public awareness activities may also include establishing patientfamily support group, as engaging with families facing similar situations can help parents of newly diagnosed patients deal with the emotional stress of a chronic disease diagnosed in their child. These factors also highlight the importance of families being supported by a professional psychologist to help them cope with the emotional stress and challenges to deal with the life-long illness of their children. Families in Pakistan often do not have access to either of these support systems, mostly available in developed countries.

This is the first survey from Pakistan to obtain specific information of factors leading to nontreatment of IMDs patients requiring FSMPs. This study provides valuable information for those in policy planning regarding service provision for patients with IMDs, in Pakistan and similar populations.

Limitations of the study: It includes a small sample size, and the call could not be recorded. Another limitation was that a telephonic survey and intellectual disability and neurological impairment assessment could not be done, requiring formal assessment using structured age-appropriate tools by a trained clinical psychologist or neuro-developmental specialist. Larger prospective, properly designed studies are needed to explore the findings of this study further.

\section{CONCLUSION}

This survey identified that non-affordability and non-prescription were the main causes resulting in the non-treatment of IMDs patients requiring FSMPs. There is a dire need for policymakers and healthcare providers to work together to address these challenges for optimal outcomes. 
Acknowledgement: We want to acknowledge Dr. Aysha Habib Khan for her support throughout this project.

\section{Conflict of interest: None.}

Source of funding: None.

\section{REFERENCES}

1. Morava E, Rahman S, Peters V, Baumgartner MR, Patterson M, Zschocke J. Quo vadis: the re-definition of "inborn metabolic diseases".JInheritMetabDis. 2015;38(6):1003-1006. doi: 10.1007/s10545-015-9893-x

2. Applegarth DA, Toone JR, Lowry RB. Incidence of inborn errors of metabolism in British Columbia, 1969-1996. Pediatrics. 2000;105(1):e10. doi: 10.1542/peds.105.1.e10

3. Roscher A, Liebl B, Fingerhut R, Olgemoller B. Prospective study of MS-MS newborn screening in Bavaria, Germany. Interim results. J Inherit Metab Dis. 2000;23:4.

4. Sherazi NA, Khan AH, Jafri L, Jamil A, Khan NA, Afroze B, et al. Selective Screening for Organic Acidurias and Amino Acidopathies in Pakistani Children. J Coll Physicians Surg Pak. 2017;27(4):218-221.

5. Hussain R, Bittles AH, Sullivan S. Consanguinity and early mortality in the Muslim populations of India and Pakistan. Am J Hum Biol. 2001;13(6):777-787. doi: 10.1002/ajhb.1124

6. Fernandes J, Saudubray JM, Van den Berghe G, Walter JH, editors. Inborn metabolic diseases: diagnosis and treatment. Springer Science \& Business Media; 2006 Nov 22.

7. Seashore MR, Friedman E, Novelly RA, Bapat V. Loss of intellectual function in children with phenylketonuria after relaxation of dietary phenylalanine restriction. Pediatrics. 1985;75(2):226-232.

8. Camp KM, Lloyd-Puryear MA, Huntington KL. Nutritional treatment for inborn errors of metabolism indications, regulations, and availability of medical foods and dietary supplements using phenylketonuria as an example. Mol Genet Metab. 2012;107(1):3-9. doi: 10.1016/j.ymgme.2012.07.005

9. Acosta PJ, Grondalski RA, Liebrecht JW, Reynolds PA. Medical foods for the nutritional support of child/adult metabolic diseases. Google Patents; 1994.

10. Acosta PJ, Grondalski RA, Liebrecht JW, Reynolds PA. Medical foods for the nutritional support of infant/toddler metabolic diseases. Google Patents; 1996.

11. Baumgartner MR, Horster F, Dionisi-Vici C, Haliloglu G, Karall D, Chapman KA, et al. Proposed guidelines for the diagnosis and management of methylmalonic and propionic acidemia. Orphanet J Rare Dis. 2014;9:130. doi: 10.1186/s13023-014-0130-8

12. Wilcken B. An Introduction to Nutritional Treatment in Inborn Errors of Metabolism-Different Disorders, Different Approaches. Southeast Asian J Trop Med Public Health. 2004;34:198-201.

13. Stippler D, Bode V, Fischer M, Kollex K, Rohde E, Tisowsky $\mathrm{B}$, et al. Proposal for a new practicable categorization system for food for special medical purposes - Enteral nutritional products. Clin Nutr ESPEN. 2015;10(6):e219-e223. doi: 10.1016/j.clnesp.2015.07.003

14. Hafeez A, Ijaz A, Chaudhry N, Ali O, Khadim MT. Diagnosis of inherited metabolic disorders by selective metabolite testing: three years experience at a tertiary care center in Rawalpindi. J Pak Med Assoc. 2020;70:53-57. doi: 10.5455/ JPMA.301908
15. Cheema HA, Malik HS, Parkash A, Fayyaz Z. Spectrum of inherited metabolic disorders in Pakistani children presenting at a tertiary care centre. J Coll Physicians Surg Pak. 2016;26(6):498-502.

16. Hafeez A, Hayat A, Hafeez J, Malik SS, Khadim MT, Ahmed N,etal. Two years experience of analytical and diagnostic challenges in urine organic acid analysis on gas chromatography-mass spectrometry. Pak Armed Forces Med J. 2018;68(5):1133-1137.

17. Mansoor, S. Trends of congenital hypothyroidism and inborn errors of metabolism in Pakistan. Orphanet J Rare Dis. 2020;15(1):1-10. doi: 10.1186/s13023-020-01602-6

18. Ehtizaz L, Haider S, Mahmood Z, Nazir S, Tehseen T, Mahmood T. Spectrum of Inherited Metabolic Diseases in neonates and children presenting at Izzat Ali Shah Hospital, Wah Cantt. J Rawal Med Coll. 2021;25(1):72-76.

19. Afroze B, Lakhani L, Naz F, Somani S, Yunus ZM, Brown $\mathrm{N}$, etal. Challenges identified in the management of patients with inherited metabolic disorders-A five year experience from Pakistan. Egypt J Med Hum Genet. 2016;17(3):259-264. doi: 10.1016/j.ejmhg.2016.03.002

20. Majid H, Khan AH, Jafri L, Jamil A, Yousufzai N, Fatima M, etal. Diagnostic dilemma of patients with methylmalonic aciduria: Experience from a tertiary care centre in Pakistan. J Pak Med Assoc. 2018;68(4):510.

21. Ahmed S, Majid H, Jafri L, Khan AH, Ali ZZ, Afroze B, etal Retrospective study of patients with hyperphenylalaninemia: Experience from a tertiary care center in Pakistan. J Pak Med Assoc. 2019;69(4):509.

22. Thatte U, Hussain S, Rosas-Valera D, Malik MA. EvidenceBased Decision on Medical Technologies in Asia Pacific: Experiences from India, Malaysia, Philippines, and Pakistan. Value in Health. 2009;12(s3). doi: 10.1111/j.15244733.2009.00622.x

23. Berry SA, Kenney MK, Harris KB, Singh RH, Cameron CA, Kraszewski JN, et al. Insurance coverage of medical foods for treatment of inherited metabolic disorders. Genet Med. 2013;15(12):978-982. doi: 10.1038/gim.2013.46

24. Mlcoch T, Puda R, Jesina P, Lhotakova M, Sterbova S, Dolezal T, etal. Dietary patterns, cost and compliance with low-protein diet of phenylketonuria and other inherited metabolic diseases. Eur J Clin Nutr. 2018;72(1):87-92. doi: 10.1038/ ejcn.2017.102

\section{Authors Contribution:}

HM collected, analyzed the data and prepared the manuscript.

LJ critically reviewed the manuscript for intellectual content.

ZZA conducted the telephonic survey, entered data and reviewed manuscript.

BA conceived the idea, supervised the project and critically reviewed manuscript for intellectual content and responsible for the accuracy or integrity of study.

All authors reviewed the final manuscript and agreed with its submission to PJMS. 